\title{
Is Comprehensive Two-Dimensional Gas Chromatography Here to Stay?
}

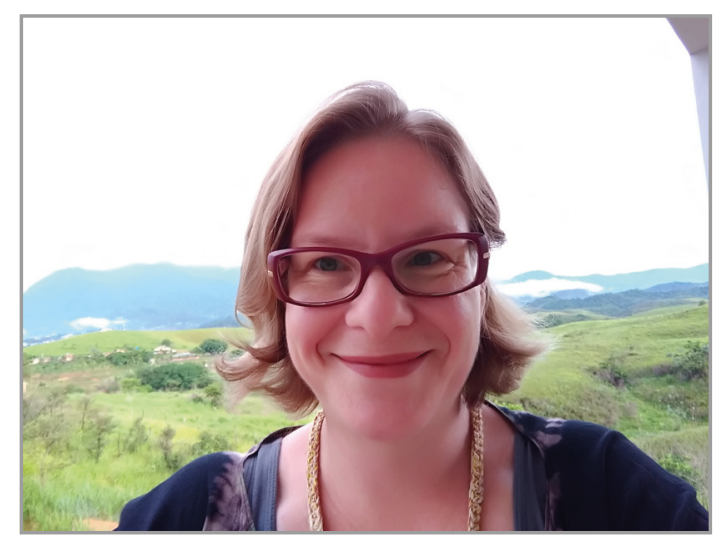

Carin von Mühlen $\triangle$

Associate Professor

Chemistry and Environmental Department

Faculty of Technology at Rio de Janeiro State University

Resende Regional Campus, Resende, RJ, Brazil

Gas chromatography is the most established analytical technique to investigate volatile and semivolatile organic compounds in real life samples. Since its onset, one of the most pursued challenges of this technique has been to work with very complex samples associated with high throughput analysis. In 1991, one important analytical tool was added to this family: comprehensive two-dimensional gas chromatography $(G C \times G C)$. With this technique, in its variable configurations, it is possible to separate thousands of substances in one unique chromatographic run. The association of this technique with fast data acquisition rate detectors, such as time-of-flight mass spectrometers, and, recently, highresolution mass spectrometers, has added the analytical power needed to elegantly identify organic compounds in very complex samples.

There are more than 1.4 thousand papers published in Science Direct records in 2019 alone with $\mathrm{GC} \times \mathrm{GC}$, which is close to $6 \%$ of publications in gas chromatography. Twenty years ago, GC×GC was responsible for less than $2 \%$ of publications. The question that arises is why this technique is not being used everywhere. Why it is growing so slowly? What are people afraid of? Are there specific difficulties associated with it?

We can think of possible answers to these questions. The first resistance of chromatographers to jumping to $G C \times G C$ may be unfamiliarity with a different way of performing chromatography and analyzing data. When dealing with two columns with different internal diameters and stationary phases coupled together, you need to revisit the optimization of parameters such as the injection speed in your method, flow rates, column temperatures and other parameters that improve your separation. Chromatograms are now tridimensional, and how to interpret and group thousands of peaks is another challenge. Once you get used to it, you become familiar with it, but this knowledge is not straightforward.

The second resistance is related to instrumentation costs and operation. While commercial GC $\times G C$ equipment is considerably more expensive than traditional chromatographs, the home-made systems are limited by the data processing systems available. Some modulators need cryogenic liquids, such as liquid nitrogen or $\mathrm{CO}_{2}$, for their operation, which increases the costs and logistics for operation. The third resistance is related to the way the technique has been presented in the literature. As a frontier technique with high resolving power, most publications presented only qualitative results, leading the reader to conclude that this technique is not recommended for quantitative work or for fast throughput analysis. On top of that, different column configurations have been tested in several publications directing the chromatographers to consider these studies as a mandatory step for method optimization. This could be true in the earlier publications, but once the technique was established, method optimization became as easy as one-dimensional chromatographic methods. 
As a rising technology, authors are starting to push the technique to its limit, demonstrating high throughput quantitative work and the limits of its resolution power. Instrumentation is becoming less expensive, and liquid cryogenic-free modulators are growing in importance. Software is becoming user friendly, with data set volumes easier to handle. Consequently, I believe that in the near future the GC $\times G C$ will occupy the prominent position it deserves in the history of chromatography. In my opinion, this technique is here to stay.

\section{REFERENCE}

Liu, Z. Y.; Phillips, J. B. J. Chromatogr. Sci., 1991, 29, pp 227-234 (https://doi.org/10.1093/ chromsci/29.6.227). 